\title{
Computational analysis of transitional air flow through packed columns of spheres using the finite volume technique
}

\author{
M.J Baker* \\ G.R Tabor* \\ School of Engineering, Mathematics and Physical Sciemces (SEMPS), Harrison Building, \\ University of Exeter, North Park Road, Exeter EX4 $4 Q F, U K$
}

\begin{abstract}
We compare computational simulations of the flow of air through a packed column containing spherical particles with experimental and theoretical results for equivalent beds. The column contained 160 spherical particles at an aspect ratio $N=7.14$, and the experiments and simulations were carried out at particle Reynolds numbers of $\left(R e_{d p}=700-5000\right)$. Experimental measurements were taken of the pressure drop across the column and compared with the correlation of Reichelt (1972) using the fitted coefficients of Eisfeld and Schnitzlein (2001). An equivalent computational domain was prepared using Monte Carlo packing, from which computational meshes were generated and analysed in detail. Computational Fluid Dynamics calculations of the air flow through the simulated bed was then performed using the finite volume technique. Results for pressure drop across the column were found to correlate strongly with the experimental data and the literature correlation. The flow structure through the bed was also analysed in detail.
\end{abstract}

Key words: Packed Bed,Porous media, Fluid mechanics, Computational fluid dynamics, Simulation

\footnotetext{
${ }^{*}$ Corresponding author

Email addresses: mb304@ex.ac.uk (M.J Baker), grtabor@ex.ac.uk (G.R Tabor)
} 


\section{Introduction}

Packed columns and packed beds are used extensively in industry for absorption, stripping and distillation operations (Perry and Green, 1997). As a result there has been a great deal of interest in analysing their behaviour (Sahimi, 1994), particularly in regard to understanding the physical processes involved (Adler, 1992). A packed bed is a volume of space filled with a packing material, which can be random in size and shape, such as gravel or charcoal, or comprise uniform geometric shapes such as spheres or cylinders. A packed column is a special case where the bed is contained in a tube or other narrow cylindrical vessel. When a fluid passes through a packed bed, it percolates through a network of voids or channels and interacts with the bed particles, creating a drop in pressure. Due to this, a packed bed is generally characterised by a number of key parameters such as porosity, particle size and bed depth. In the case of catalysts and other industrial processes, bed surface area may also be a key parameter in achieving a maximum fluid-to-particle interaction. Packed columns possess similar attributes, but the flow is more complex due to boundary effects at the walls of the vessel; the presence of the wall alters the flow both directly and because it upsets the packing of the particles within the column.

In early work in this area, researchers (Blake, 1922; Kozeny, 1927; Burke and Plummer, 1928; Carman, 1937; Ergun, 1952) studied the influential parameters of packed beds and columns, and through experiments and statistical methods developed a set of equations based around theoretical tube models. These formulate pressure drop per unit length as a function of the velocity, but do not take into account the large scale bed geometry, and therefore assume the bed is homogeneous. Bed geometry is a key aspect to understanding how a fluid percolates through a bed, with regard to which routes it takes, volumes where the flow is more or less uninhibited, and the role of stagnation zones. Identifying and examining these aspects of the flow is best achieved using Computational Fluid Dynamics (CFD). Such work has been carried out out on small numbers of spheres, with low particle to tube ratios; however the computational cost of 
full simulation of the microstructural flow on larger beds has meant that this is only just becoming a realistic approach.

In most applications of CFD to packed columns the ratio of particle to tube diameter (aspect ratio, $N=D / d_{p}$ ) is low. This is attributed to the complex geometries associated with high aspect ratio beds and the associated problems of generating the geometry and discretising it as a mesh. Naturally, in the early days of computing the only beds which could be practically analysed were those of low aspect ratios in cases containing only a couple of particles. As computers advanced and computational power became more readily available, the number of particles being modelled grew considerably. Spherical particles were generally chosen due to the possible regular packing regimes, which can easily be determined mathematically. In addition, spherical particles only require a single coordinate, e.g cartesian or polar, and single value of radius to determine the particle's location and geometric properties.

Some of the earliest work applying CFD to packed beds was that of Dalman et al. (1986). They used 2D CFD simulations to investigate flow structure and heat transfer in an axisymmetric radial plane, and a velocity-pressure formulation of the Navier-Stokes equations solved numerically using a finite element technique. They investigated laminar flow at Reynolds numbers up to $R e=200$, with Prandtl number of 0.72 and 7.0, for a range of sphere sizes and separations. Although the packing possibilities are limited using this approach, the work gave a valuable insight into the flow structure through the bed and indicated the formation of eddies between the bed particles (Taskin, 2007). Lloyd and Boehm (1994) studied flow and heat transfer around a linear array of 8 spheres using the finite element package FIDAP. They used Reynolds number of 40, 80 and 120 with Prandtl numbers ranging from 0.73-7.3. This work was a considerable advancement from that of Dalman et al. (1986) due to the number of particles being analysed. Similar to the work of Derx and Dixon (1996) it was possibly one of the first $3 \mathrm{~d}$ studies performed on an array of spheres. Logtenberg and Dixon (1998) modelled a bed of 8 spheres in the form of two layers of four spheres perpendicular to the flow with low aspect ratios of 2.43. They used the 
commercial CFD code FLOTRAN to investigate fluid flow h eat transfer using air for Reynolds numbers of $R e=9-1450$. Logtenberg et al. (1999) modelled a bed of ten spheres with aspect ratios of $N=2.43$ using FEM. Similar to the earlier work of Lloyd and Boehm (1994) they focussed on heat transfer and fluid flow, focusing on wall-particle contact points.

For larger geometries one possible approach is to assume a regular structure to the bed which can be reproduced using a simple unit cell (Hellström and Lundström, 2006). An example of this was presented by Tóbis (2000) using a simple cubic unit cell and comparing against theoretical expectations and experiments on similar structured beds. In this work they used the finite volume code Fluent and the standard $k-\varepsilon$ turbulence model to investigate flow around the particles in detail. A similar approach was used by Calis et al. (2001) to model a limited subset of bed geometries known as composite structured packed beds; instead of a single unit cell they represented the bed in terms of a single linear row of spheres with appropriate boundary conditions. Manjhi et al. (2006) examine regular packings of small numbers of spheres $(<10)$ in a cylindrical tube using lattice Boltzmann techniques, and were able to produce convincing visualisations of the microstructural flow in these highly simplified geometries. Attempts have also been made to blend numerical simulations on simplified geometries with empirical data (Tobís, 2008). Dixon and Nijemeisland (2001) used the finite volume technique to model small clusters of structured spheres with aspect ratios $N=2$ (Reynolds numbers of $R e=373-1922$ ) and $N=4$ $(R e=180)$. In the case of the higher aspect ratio bed $(N=4)$ the mesh is too coarse to determine an accurate flow field, however it produced convincing results for the other aspect ratio $(N=2)$. The limiting factor on all previous packed beds CFD work is determining a geometry of a sufficient size to give realistic results.

The task of reproducing a genuine random bed geometry is more challenging, but not impossible. One approach is to use $3 \mathrm{~d}$ scanning techniques such as MRI or micro-CT to scan the bed; image-based meshing techniques originally developed in the field of computational biomechanics can then be used to recon- 
struct the bed geometry with a fair degree of accuracy. This sort of approach has been applied to probe the flow in various porous media, for instance in porous foams (Tabor et al., 2008) as well as other aspects of microstructure (Youssef et al., 2005). The alternative is to construct an entirely artificial bed geometry via some form of randomised object packing algorithm. Caulkin et al. (2007) describe the code DigiPac, where the objects to be packed are represented as assemblages of voxels in space which are allowed to move one grid spacing at a time within a cubic lattice, and their resulting motion allows them to explore every possible packing space. Earlier work on packing thick fibres lead to the development of the code MacroPac (Evans, 1988; Rowe et al., 2005), which uses a Monte Carlo approach to explore packing space. Once the packing has been determined the task is then to mesh the geometry using some appropriate meshing algorithm, and then apply CFD to the resulting domain. Zeiser et al. (2002) apply Lattice Boltzmann techniques to solve the flow in a packed column. Their tube-to-particle-diameter ratio is low $\left(D / d_{P}=3\right.$, where $D$ is the tube diameter and $d_{P}$ the sphere diameter) so wall effects are dominant in their flow; however they are able to investigate in some detail the microstructural flow in the bed. Pan et al. (2006) apply Lattice Boltzmann techniques to both a simple bcc unit cell model and also to a random bed; the focus of their paper is comparing different variants of the Lattice Boltzmann technique rather than experimental validation. However, there are still unresolved computational concerns with LBM due to its restrictions to a limited class of mesh, and other approaches to solving the Navier-Stokes equations are still appropriate (Tabor et al., 2008). Finally, Magnico (2003) uses finite volume techniques applied to a somewhat larger bed (several hundred particles and $D / d_{P}$ of $5-7$ ) but quite a low Reynolds number (between 20 and 200).

In this paper we use MacroPac to generate the sphere packing using the Monte Carlo approach, with the resulting sphere centres being exported to the CAD package AutoCAD to recreate the spheres. The resulting geometry is then imported into the commercial mesh generator Gambit, and meshed using a fine tetrahedral mesh. This mesh is then imported into the finite volume 
CFD code Fluent for solution. At the same time, experimental measurements were performed on an equivalent bed of spheres (marbles) of equivalent size. Measurements of the pressure drop for air flow through the bed were taken and correlated against theoretical expectation of Reichelt (1972), Eisfeld and Schnitzlein (2001) and against the CFD results. Our work differs from the earlier work described above as it is at a higher Reynolds number than that of Magnico (2003) and a larger bed than that of Zeiser et al. (2002); Dixon and Nijemeisland (2001). As with the work of Tóbis (2000), we use the CFD code Fluent but with the $k-\omega$ turbulence model, and use a random packing of 160 spherical particles as an alternative to a simple unit cell or a regular packing. Comparing against our own experiments rather than against results from the literature (however good), also enables us to match exactly the size of particles and column and the flow conditions.

\subsection{Theoretical Background}

Of interest for the flow though packed beds is the relationship between flow velocity or Reynolds number and the drop in pressure across the bed. Many theoretical correlations exist to calculate this, such as that reported by Blake (1922); Kozeny (1927) for laminar flow and by Burke and Plummer (1928); Carman (1937) for turbulent flow. Hicks (1970) proposed a new equation which fits the data of Ergun (1952), Handley and Heggs (1968) and Wentz and Thodos (1963). In many applications, applicable to both laminar and turbulent flow, the well established Ergun equation (Ergun, 1952; Ergun and Orning, 1949) is used calculate pressure drop through a packed bed given as

$$
\frac{\Delta P}{L}=\frac{150 \mu(1-\varepsilon)^{2} U_{S}}{\varepsilon^{3} d_{P}^{2}}+\frac{1.75(1-\varepsilon) \rho U_{S}^{2}}{\varepsilon^{3} d_{P}}
$$

where $\varepsilon$ is bed porosity, $U_{S}$ is superficial velocity (the equivalent velocity if the bed was not there) and $d_{P}$ is particle equivalent diameter given in the form

$$
d_{P}=\frac{6 V_{P}}{S_{A}}
$$

with $V_{P}$ the particle volume and $S_{A}$ its surface area. The Ergun equation is a model to determine the expected behaviour of a packed bed, up until the point 
of fluidisation, and is heavily dependent on particle diameter and bed porosity, where the porosity is given as the ratio of the void volume to the total volume $\left(\varepsilon=V_{v} / V\right)$. The particle equivalent diameter is a way of introducing the shape of the particle into this; for spherical particles it is just the sphere diameter. The numerical coefficients for the Ergun equation are usually given as 150 and 1.75 (Ergun, 1952). Leva (1959) suggested alternative coefficients of 200 and 1.75, with MacDonald et al. (1979) recommending coefficients in the range of 180 and 1.8 - 4.0. Du Plessis and Woudberg state that the coefficients are severely dependant on the interstitial physical flow conditions that vary considerably between different types of structure (Plessis and Woudberg, 2008).

The Ergun equation is strictly applicable only for infinite beds; it takes no account of boundary effects. To determine the applicability of the Ergun equation we use the bed aspect ratio $N=D / d_{p}$ which compares the tube diameter $D$ to the particle diameter $d_{p}$. Choi et al. (2008) suggest that when the bed diameter $(D)$ is not significantly larger than the particle diameter $\left(d_{P}\right)$, the flow maldistributions and wall friction are not negligible. Similarly, Foumeny et al. (1993) suggest that when the aspect ratio is $<50$, wall effects cause the Ergun equation to yield a poor result. Choi et al. (2008) go on to suggest that for low Reynolds numbers $(R e /(1-\varepsilon)<10)$ the Ergun equation tends to under predict the pressure drop and in the larger Reynolds regime $(R e /(1-\varepsilon)>10)$ the Ergun equation overpredicts the pressure drop in comparison to experimental results. Many correlations have been published to account for the confining wall, such as those proposed by Choi et al. (2008) and Gibilaro (2004). One of the most promising correlations was proposed by Reichelt (1972) and further improved by Eisfeld and Schnitzlein (2001). Eisfeld and Schnitzlein (2001) take a comprehensive database of more than 2300 experimental data points from 24 published experimental studies and plot and determine values for the the coefficients $\left(K_{1}, k_{1}, k_{2}\right)$ for the Reichelt (1972) equation.

In this paper we report our experimental and computational results in the 
form of dimensionless pressure drop, defined as

$$
\phi=\frac{\Delta P}{\rho_{0} U_{S}^{2}} \frac{d_{p}}{L}
$$

We define the particle Reynolds number $R e_{d p}$ as

$$
R e_{d P}=\frac{\rho U_{S} d_{P}}{\mu}
$$

which defines the flow regime, where the flow can be characterised as laminar $\left(\operatorname{Re}_{d P}<10\right)$, transitional $\left(10<\operatorname{Re}_{d P}<300\right)$ or turbulent $\left(\operatorname{Re}_{d P}>300\right) \mathrm{Zi}-$ olkowska and Ziolkowska (1988). Carman (1937) suggest that in laminar flow, the dimensionless pressure drop $\phi$ is proportional to $1 / R e_{d p}$. Here, we compare both experimental and computational results with the correlation described by Reichelt (1972) using the fitted coefficients determined by Eisfeld and Schnitzlein (2001) as

$$
\phi=\frac{K_{1} A_{W}^{2}}{R e_{d p}} \frac{(1-\varepsilon)}{\varepsilon^{3}}+\frac{A_{W}}{B_{W}} \frac{1-\varepsilon}{\varepsilon^{3}}
$$

where $K_{1}=154$ (Eisfeld and Schnitzlein, 2001)

$$
A_{W}=1+\frac{2}{3\left(D / d_{p}\right)(1-\varepsilon)}
$$

and

$$
B_{w}=\left[k_{1}\left(\frac{d_{p}}{D}\right)^{2}+k_{2}\right]^{2}
$$

where $k_{1}$ and $k_{2}$ are given as 1.15 and 0.87 respectively (Eisfeld and Schnitzlein, 2001)

\section{Method}

\subsection{Experimental Setup}

The experimental setup used in this work requires methods for measuring values of pressure difference $\Delta P$, and volumetric flow rate $(Q)$ and hence velocity $(U)$ and Reynolds number $(R e)$. The rig is based on that of Tóbis (2000), with the addition of pressure taps into the bed at $0.1 \mathrm{~m}$ vertical intervals, as shown in figure $1.14 \mathrm{~mm}$ marbles were used as the spherical particles for the bed. The 
packed bed was supported by a wire mesh screen enclosed in a $100 \mathrm{~mm}$ diameter Perspex column, with a length of $300 \mathrm{~mm}$. Air was forced through the bed using a centrifugal pump. Volumetric flow rate $Q$, and hence average velocity $U$, was determined by measuring differential pressure $(\Delta P)$, across a plate orifice manufactured from Perspex. An experimental value for the flow coefficient $C_{f}$ of the plate orifice was calibrated against a velocity profile determined by hot-wire anemometers attached to a data logger.

The plate orifice method was chosen over that of a Pitot static tube, because the Pitot static tube only determines the velocity at its immediate location, meaning that a full flow profile using this method would require many readings to be taken in different locations across the pipe section, then averaged to reduce error. One disadvantage of using a plate orifice is that there is a significant pressure drop immediately after the orifice; to reduce this, a Venturi meter could be used as an alternative, where the drop in pressure due to the device is less significant. However, due to the inexpensiveness and ease of manufacture, along with general accuracy and ease of calibration, the benefits of using plate orifice outweigh the disadvantages.

Experimental pressure drop in this work was calculated by means of a differential micro-manometer attached to a data logger. The data logger readings were averaged to reduce error from small fluctuations in pressure due to turbulence, with differential pressures taken from immediately before the bed (0.0) and at $0.1 \mathrm{~m}$ intervals. To validate the total pressure drop, differential pressure was recorded between intervals and added together, then compared with total pressure drop.

An accurate value of air density was determined by using a barometer to determine atmospheric pressure $p$ and a $\mathrm{k}$ type thermocouple placed in the flow to determine flow temperature $T$. This was used in conjunction with the universal gas constant $R=287 \mathrm{~J} / \mathrm{kgK}$, to determine an air density of $1.117 \mathrm{~kg} / \mathrm{m}^{3}$. 


\subsection{Computational Packing of Spheres}

For this work, the particle packing simulation program MacroPac (Intelligensys, UK) was used to generate packed beds of spheres of the same diameter as those used for the real experiment. In MacroPac the Monte Carlo method is used to pack the spheres to the maximum packing possible, by simulated shaking of the column and packing medium. Characteristic parameters of the packing such as porosity $(\varepsilon=0.48)$, number of bed particles (164) and contacts can also be determined with this program. The container geometry used as an input parameter for MacroPac was identical to that of the experiment.

Having generated these computational beds, the coordinates of the sphere centres were exported to the $\mathrm{CAD}$ package AutoCAD and used to regenerate the packed spheres in ACIS format for export to the automatic meshing program Gambit. Figure 2 shows a view of the spheres in the bed displayed in Fluent. Although relatively simple, using this technique there is scope to produce much larger beds of spherical media, the only limitation is the large amount of computational power required in mesh generation and computational simulation.

\subsection{Computational Fluid Dynamics (CFD)}

Computational Fluid Dynamics is the use of computers to solve fluid flow problems. Conventionally it is usually taken to mean the solution of the governing equations, the Navier-Stokes equations in 2 or 3 dimensions for laminar flow, or the averaged Navier-Stokes equations together with a turbulence model in the case of a turbulent flow. For a laminar flow then, the equations being solved are as follows :

$$
\begin{aligned}
\nabla \cdot \underline{u} & =0 \\
\frac{\partial \underline{u}}{\partial t}+\nabla \cdot \underline{u} \underline{u} & =-\frac{1}{\rho} \nabla p+\nu \nabla^{2} \underline{u}
\end{aligned}
$$

For a turbulent flow, an averaging process is applied to the equations. Most often this is an ensemble or time averaging, known as Reynolds averaging, which 
generates the Reynolds Averaged Navier-Stokes (RANS) equations :

$$
\begin{aligned}
\nabla \cdot \underline{\widehat{u}} & =0 \\
\frac{\partial \underline{\widehat{u}}}{\partial t}+\nabla \cdot \underline{\underline{u}} \underline{\underline{u}}+\nabla \cdot \underline{\underline{R}} & =-\frac{1}{\rho} \nabla p+\nu \nabla^{2} \underline{\widehat{u}}
\end{aligned}
$$

where ^ denotes a Reynolds-averaged flow variable, and $\underline{\underline{R}}$ is an additional term called the Reynolds Stress, which represents the effect of the turbulence (which has been removed by the averaging procedure) on the mean flow. This is an unknown quantity, and so further equations must be introduced to close the set of equations; these are referred to as turbulence models.

Various turbulence models have been proposed to complete the set of equations. The most frequently used models are those based on the Boussinesq eddy viscosity approach, in which the effect of the turbulence is taken to be equivalent to the enhancement of the viscosity of the fluid, so $\underline{\underline{R}}$ is modelled as $\nu_{t} \nabla^{2} \underline{\widehat{u}}-\frac{2}{3} k I$. where $k$ is the turbulent kinetic energy and $\nu_{t}$ the turbulent viscosity. In turn, the turbulent viscosity $\nu_{t}$ is modelled in terms of two additional variables; the turbulent kinetic energy $k$ and the turbulent dissipation $\epsilon$, for which transport equations can be formulated and solved. This leads to the well-known $k-\epsilon$ family of turbulence models, which are extensively used in CFD (and have been applied to this problem by Tóbis (2000)). An alternative to this which has found favour recently is the $k-\omega$ turbulence model, where the turbulent kinetic energy $k$ is paired with the turbulent frequency $\omega=\epsilon / k$, obtained from the following set of partial differential equations;

$$
\frac{\partial}{\partial t}(\rho \kappa)+\frac{\partial}{\partial x_{i}}\left(\rho \kappa u_{i}\right)=\frac{\partial}{\partial x_{j}}\left(\Gamma_{\kappa} \frac{\partial_{\Gamma}}{\partial x_{j}}\right)+G_{\kappa}-Y_{\kappa}+S_{\kappa}
$$

and

$$
\frac{\partial}{\partial t}(\rho \omega)+\frac{\partial}{\partial x_{i}}\left(\rho \omega u_{i}\right)=\frac{\partial}{\partial x_{j}}\left(\Gamma_{\omega} \frac{\partial_{\Gamma}}{\partial x_{j}}\right)+G_{\omega}-Y_{\omega}+S_{\omega}
$$

where $G_{\kappa}$ is turbulent kinetic energy generation as a result of the average gradients in velocity, $G_{\omega}$ is the specific dissipation rate. $\Gamma_{\kappa}$ and $\Gamma_{\omega}$ are the effective diffusivity. $S_{\kappa}$ and $S_{\omega}$ are source terms. $Y_{\kappa}$ and $Y_{\omega}$ represent the turbulent dissipation of $\kappa$ and $\omega$. $\omega$ exhibits much nicer behaviour near walls than $\epsilon$ and 
so this model is preferred for low Reynolds number turbulence. Since the flow in our simulation is likely to be transitional to turbulent we have adopted the $k-\omega$ model with Fluent's enhanced wall treatment.

We solve the governing equations (9) using the finite volume approach. In this, the domain of interest is divided into numerous small cells, and the governing equations integrated across the volume of each cell. Gauss' theorem is used to convert the spatial derivative term $\nabla .(\underline{u} \underline{u})$ into a surface integral of the flux across the faces bounding the cell, which converts the governing equations into a set of difference equations which can be solved numerically. For this we use the commercial CFD code Fluent; interpolation from cell centre to cell face is carried out using 2nd order upwind differencing for the momentum equation, and the non-linear nature of the equations is treated iteratively, using PISO for transient calculations and SIMPLE for steady state calculations. The SIMPLE algorithm (semi-implicit method for linked equations) is effectively a guess and correct procedure for the calculation of pressure developed by S.V.Patankar and D.B.Spalding (1972) (Versteeg and Malalasekera, 1995).The algorithm PISO, which stands for pressure implicit with splitting of operators, involves a single predictor step and two corrector steps (Versteeg and Malalasekera, 1995). Essentially the PISO algorithm is a more advanced SIMPLE algorithm with the inclusion of an extra corrector step for enhancement(Versteeg and Malalasekera, 1995). To ensure stability, simulations were run initially using a steady state solver and then using this as a basis progressing to an unsteady solver. Courant numbers were kept low $\left(1 \times 10^{-8}\right)$ to ensure stability. Convergence to a solution was judged based on the mass flow rate at the outlet of the domain.

\section{Computational domain}

The complex geometrical properties of random packed beds makes it difficult to produce a valid mesh. Even when a mesh has been produced the quality is often dubious containing non-positive volumes and highly skewed elements, which often lead to simulation divergence and instability with no solution convergence. 


\begin{tabular}{|c|c|}
\hline Properties & Mesh \\
\hline Max Cell vol & $1.834 \mathrm{~mm}^{3}$ \\
Min Cell vol & $5.208 \times 10^{-3} \mathrm{~mm}^{3}$ \\
Max face area & $3.14 \mathrm{~mm}^{2}$ \\
Min face area & $1.987 \times 10^{-2} \mathrm{~mm}^{2}$ \\
Mesh Volume & $5.796 \times 10^{5} \mathrm{~mm}^{3}$ \\
\hline
\end{tabular}

Table 1: Mesh data

In this work the mesh creation was done using Gambit, a general-purpose program providing a variety of automatic meshing strategies. Here the domain was meshed using the Tet/hybrid and TGrid options; this generates meshes comprised predominantly of tetrahedral cells but includes other cells (prismatic and hexahedral) where necessary. Surfaces were not meshed separately and so were automatically meshed as triangles. In this work the domain was split into $1,168,328$ cells containing $2,456,980$ faces and 248,950 nodes. The grid was then partitioned along the principal axis into 4 segments to allow paralization of the domain to reduce convergence time and run on a Beowulf cluster; each node of the cluster comprises two dual-core $2.6 \mathrm{GHz}$ processors and $8 \mathrm{~GB}$ RAM. To reach an overall solution required between 4 and 8 computer hours.

To capture the full effects of viscous drag for microstructural flow the mesh must be fine enough. To investigate this it is necessary to conduct a mesh convergence study, by increasing the number of mesh elements and monitoring the pressure at the model pressure outlet. In this case the study (figure 4) demonstrated that the results did not significantly change after 1,168,328 cells; a mesh finer than this would yield no advantage in producing more accurate results and would be computationally more expensive.

The quality of the mesh was analysed using the dimensionless parameter equivolume skew $\left(E_{V}\right)$, given by

$$
E_{V}=\frac{V_{O}-V_{C}}{V_{O}}
$$


where the $V_{O}$ is the optimal cell size of an equilateral cell with the same circumradii and $V_{C}$ is the actual cell size. A cell equivolume skew of 0 indicates a "perfect" cell whilst a value of 1 indicates a highly skewed, completely degenerate element. From figure 5 it can observed that the mesh contains a few highly skewed elements. When meshing complex spherical geometries, such as this, skewed cells are often unavoidable. In the literature, for the case of regular sphere packings, particle distances from one another (gaps) can be set to allow a suitable size element to be fitted. In our case distances vary quite considerably and this cannot be done. The majority of skewed elements are located in areas of particle near contact points. Fluent provides a range of features to smooth the mesh and reduce skewness after the mesh has been produced. However this does not completely eliminate all skewed elements, and these cause problems with the numerical solution of the flow. Fluent also implements skewness correction and neighbour coupling as part of the PISO loop, which help to ameliorate the problem. In this case the neighbour correction is set to 1 and the skewness correction to 6 , to stabilise the solution. For this work Fluent's enhanced wall treatment is adopted in which case to resolve the laminar sub-layer $y+$ values for the wall adjacent cell need to be around $y+=1$. Figure 6 demonstrates that this has been achieved for most near-wall cells.

\section{Results}

Figure 7.a. shows our raw results, comparing computed values of pressure drop per unit length $d p / L$ against particle Reynolds number $R e_{p}$ (circles) with our experimental results (triangles) and empirical correlations (Eisfeld and Schnitzlein (2001)). Calculated values of $d p / L$ were evaluated by taking areaweighted averages of the pressure at the top and bottom of the bed. Figure 7.b. shows the same information but with the pressure drop expressed in terms of $\phi$ using equation 3, which is a standard way of presenting the information in the literature. To demonstrate the variation of pressure through the bed, figure 8.a. presents the area-averaged pressure on regularly-spaced slices through the bed. 
Regression analysis has been used to fit straight lines through the data sets; this clearly shows the linear relationship between pressure and height through the domain.

MacroPac provides the facility to evaluate porosities across the bed. Results for this bed are shown in figure 8.b. As expected the porosity is roughly uniform across the core region but rises within the near-wall region of the column where the packing is affected by the presence of the wall. Some low aspect ratio two zone correlations such as DiFelice and Gibilaro (2004) rely on two values of porosity, usually defined as wall and core porosities. Using MacroPac's porosity functionality these can be determined by the averaging the values of the wall region (1 sphere radius), and the core region.

The actual flow patterns through the beds are shown in figure 9. These show colour plots of velocity magnitude and turbulent kinetic energy on vertical and horizontal cutting planes through the domain. The random packing of the spheres is clearly visible; also note the practical difference between the interior of the bed and the near-wall region where the packing of the spheres is constrained by the presence of the wall. This lowers the porosity in these regions and a significant fraction of the flow is being channelled through this region. One advantage of computational simulation of the flow is the sheer volume of data available for analysis; in particular here, flow velocities at every point in the bed, which are available for statistical analysis.

\section{Discussion}

As expected CFD and experimental data show a strong correlation, with the CFD marginally overpredicting the drop in pressure (figure 7.a,b). Both CFD and experimental match theoretical results closely with the experimental and CFD slightly underpredicting the expected curve from Reichelt (1972); Eisfeld and Schnitzlein (2001). The small discrepancy between the results of the CFD, experimental and the correlation proposed by Reichelt (1972) can be attributed to the fact that the coefficients determined by Eisfeld and Schnitzlein (2001) 
are taken from a data set of 2300 data points from 24 sets of published results over a timescale of 70 years, for a range of subtly different experimental cases. Thus it represents an average of results which themselves exhibit quite a spread of values; the spread being due to factors which have not been controlled for. None the less, they correlate well and in this case we are comparing our CFD with equivalent beds of the same aspect ratio and Reynolds number, whereas the pressure drop data analysed by Eisfeld and Schnitzlein (2001) is over an extensive range of Reynolds number and aspect ratios.

Near-wall effects can also be seen in the structure of the flow patterns themselves. When fluid percolates through a network of channels, the pathway of mainstream velocity at a low Re takes the shortest route through the backbone of the bed (Andrade et al., 1997). At higher values of Re, the pathway of mainstream velocity is dictated by the bed geometry (Andrade et al., 1997). Figure 9 a-b illustrates pathways of mainstream velocity through the bed, biassed towards the wall region. This is shown for the case $R e=2771$; however the flow patterns are largely similar for all the Reynolds numbers investigated, with just the overall magnitude being altered. Similarly, figure $9 \mathrm{c}$-d plot turbulent kinetic energy $k$ on the same sections. Turbulence generation is seen to be heavily influenced by the geometry, with the complexity of the bed having a significant generation effect, whilst the near-wall region where the paths are much simpler generates much less turbulence.

\section{Conclusions}

The goal of this work was to generate and probe random packed columns of spheres using computational methods, and to demonstrate their accuracy and reliability compared to experimental and theoretical results.

The macroscopic flow behaviour, i.e. the pressure drop per unit depth, was found to correlate very well with experimental results on an equivalent bed. As expected, empirical correlations from the literature slightly overpredict the pressure drop compared with our computational and experimental results. 
Moreover the microscale flow behaviour can also be probed using this approach. Calis et al. (2001) anticipated that within five years packed beds containing a few hundred particles would be considered a 'standard' problem in terms of memory and calculation time requirements. With this achieved and as computers are becoming ever increasingly powerful, we anticipate that within a few more years it is likely that much larger beds of more than a thousand particles could be analysed in this way.

\section{References}

Adler, P. M., 1992. Porous Media: Geometry and Transport. ButterworthHeinemann, Stoneham, MA.

Andrade, J. S., Almeida, M. P., Filho, J. M., Havlin, S., Stanley, B. S. H. E., 1997. Fluid flow through porous media: the role of stagnant zones. Phys.Rev.Lett. 79 (20), 3901 - 3904.

Blake, F. C., 1922. The resistance of packing to fluid flow. Transactions of American Institute of Chemical Engineers 14, 415 - 421.

Burke, S. P., Plummer, W. B., 1928. Gas flow through packed columns. Industrial Engineering Chemistry 20, 1196-1200.

Calis, H. P. A., Nijenhuis, J., Paikert, B. C., Dautzenberg, F. M., van den Bleek, C. M., 2001. Cfd modelling and experimental validation of pressure drop and flow profile in a novel structured catalytic reactor packing. Chem.Eng.Sci. 56, $1713-1720$.

Carman, P. C., 1937. Fluid flow through a granular bed. Trans.Inst.Chem.Eng.London 15, 150 - 156.

Caulkin, R., Ahmad, A., Fairweather, M., Jia, X., Williams, R. A., 2007. An investigation of sphere packed shell-side columns using a digital packing algorithm. Computers and Chemical Engineering 31, 1715 - 1724. 
Choi, Y. S., Kim, S. J., Kim, D., 2008. A semi-empirical correlation for pressure drop in packed beds of spherical particles. Transp Porous Med 75 (2), 133 149.

Dalman, M. T., Merkin, J. H., McGreavy, C., 1986. Fluid flow and heat transfer past two spheres in a cylindrical tube. Computers and Fluids 14 (3), 267 281.

Derx, O. R., Dixon, A. G., 1996. Determination of the fixed bed wall heat transfer coefficient using computational fluid dynamics. Heat Transfer Part A $29,777-749$.

DiFelice, R., Gibilaro, L. G., 2004. Wall effects for the pressure drop in fixed beds. Chem.Eng.Sci. 59 (14), 3037 - 3040.

Dixon, A. G., Nijemeisland, M., 2001. CFD as a design tool for fixed bed reactors. Ind.Eng.Chem.Res 40, 5246 - 5254 .

Eisfeld, B., Schnitzlein, K., 2001. The influence of confining walls on the pressure drop in packed beds. Chem.Eng.Sci. 56 (14), $4321-4329$.

Ergun, S., 1952. Flow through packed columns. Chem.Eng.Prog. 48 (2), 89.

Ergun, S., Orning, A., 1949. Fluid flow through randomly packed columns and fluidised beds. Ind.Eng.Chem 41, 1179 - 1184.

Evans, K. E., 1988. The packing of thick fibres. J.Phys.D:Appl.Phys. 22, $354-$ 360 .

Foumeny, E. A., Benyahia, F., Castro, J. A. A., Moallemi, H. A., Roshani, S., 1993. Correlations of pressure drop in packed beds taking into account the effect of confining wall. Int.J.Heat Mass Trans 36, 536-540.

Gibilaro, R. D. F. L. D., 2004. Wall effects for the pressure drop in packed beds. Chemical Engineering Science 59, 3037 - 3040. 
Handley, D., Heggs, P. J., 1968. Momentum and heat transfer in regularly shaped packing. Trans. Inst. Chem. Eng 46, $251-264$.

Hellström, J. G. I., Lundström, T. S., 2006. Flow through porous media at moderate Reynolds number. In: International Scientific Colloquium: Modelling for Material Processing, Riga, June 8-9.

Hicks, R. E., 1970. Pressure drop in packed beds of spheres. Industrial and Engineering Chemistry Fundamentals 9, 500 - 502.

Kozeny, J., 1927. Ober kapillare Leitung das Wassers im Boden. S.Ber.Wiener Akad.Abt.IIa 136, 271.

Leva, M., 1959. Fluidisation. McGraw-Hill Book Company, New York.

Lloyd, B., Boehm, R., 1994. Flow and heat transfer around a linear array of spheres. Numer Heat Transfer part A 26, $237-252$.

Logtenberg, S. A., Dixon, A. G., 1998. Computational fluid dynamics of fixed bed heat transfer. Chemical Engineering Process 37, $7-21$.

Logtenberg, S. A., Nijemeisland, M., Dixon, A. G., 1999. Computational fluid dynamics simulations of fluid flow and heat transfer at the wall-particle contact points in a fixed bed reactor. Chemical Engineering Science 54, 2433 2439 .

MacDonald, T. F., El-Sayer, M. S., Mow, K., Dullen, F. A. L., 1979. Flow through porous media: The Ergun equation revisited. Industrial and Engineering Chemistry Fundamentals 18, 199-208.

Magnico, P., 2003. Hydrodynamic and transport properties of packed beds in small tube-to-sphere diameter ratio: pore scale simulation using an Eulerian and a Lagrangian approach. Chem.Eng.Sci. 58, 5005 - 5024.

Manjhi, N., Verma, N., Salem, K., Mewes, D., 2006. Simulation of 3d velocity and concentration profiles in a packed bed adsorber by Lattice Boltzmann methods. Chem.Eng.Sci. 61, 7754 - 7765. 
Pan, C., Luo, L.-S., Miller, C. T., 2006. An evaluation of Lattice Boltzmann schemes for porous medium flow simulation. Computers \& Fluids 35, 898 909.

Perry, R. H., Green, D. W., 1997. Perry's Chemical Engineers Handbook, 7th Edition. McGraw-Hill; New York.

Plessis, P. J. D., Woudberg, S., 2008. Pore-scale derivation of the ergun equation to enhance its adaptability and geralization. Chem.Eng.Sci. 63, 2576-2586.

Reichelt, W., 1972. Zur berechnung des druckverlustes einphasig durchstromter kugel- und zylinderschuttengen. Chemie-Ingenieur-Technik 44, 1068 - 1071.

Rowe, R. C., Yourk, P., Clobourn, E. A., Roskilly, S. J., 2005. The influence of pellet size, shape and distribution on capsule filling a preliminary evaluation of three-dimensional computer simulation using a monte carlo technique. International Journal of Pharmaceutics 300, 32 - 37.

Sahimi, M., 1994. Applications of Percolation Theory. Taylor \& Francis, London.

S.V.Patankar, D.B.Spalding, 1972. A calculation procedure for heat, mass and momentum transfer in 3-d parabolic flow. Int.J.Heat.Mass.Transfer 15, 1787.

Tabor, G. R., Yeo, O., Young, P. G., Laity, P., 2008. CFD simulation of flow through an open-cell foam. Int.J.Mod.Phys.C. 19 (5), $703-715$.

Taskin, M. E., 2007. CFD simulation of transport and reaction in cylindrical catalyst particles. Ph.D. thesis, Worchester Polytechnic Institute, USA.

Tóbis, J., 2000. Influence of bed geometry on its frictional resistance under turbulent flow conditions. Chem.Eng.Sci. 55, 5359 - 5366.

Tobís, J., 2008. A hybrid method of turbulent flow modelling in packings of complex geometry. Chem.Eng.Sci. 63, 2670 - 2681.

Versteeg, H. K., Malalasekera, W., 1995. An introduction to Computational Fluid Dynamics : The Finite Volume Method. Longman Scientific \& Technical. 
Wentz, C. I., Thodos, G., 1963. Total and form drag friction factors for the turbulent flow of air through packed and distended beds of spherical particles. AIChE 9, $358-361$.

Youssef, S., Maire, E., Gaertner, R., 2005. Finite element modelling of the actual structure of cellular materials determined by x-ray tomography. Acta Materialia 53, $719-730$.

Zeiser, T., Steven, M., Freund, H., Lammers, P., Brenner, G., Dusrt, F., Bernsdorf, J., 2002. Analysis of the flow field and pressure drop in fixed-bed reactors with the help of Lattice Boltzmann simulations. Phil.Trans.Roy.Soc.A: Physical and Engineering Sciences 360 (1792), 507 - 520.

Ziolkowska, I., Ziolkowska, D., 1988. Fluid flow inside packed beds. Chemical Engineering Process 23, $137-164$. 


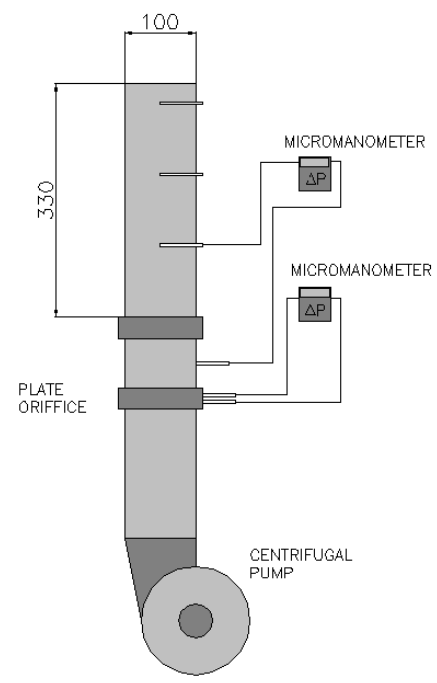

Figure 1: Experimental setup 


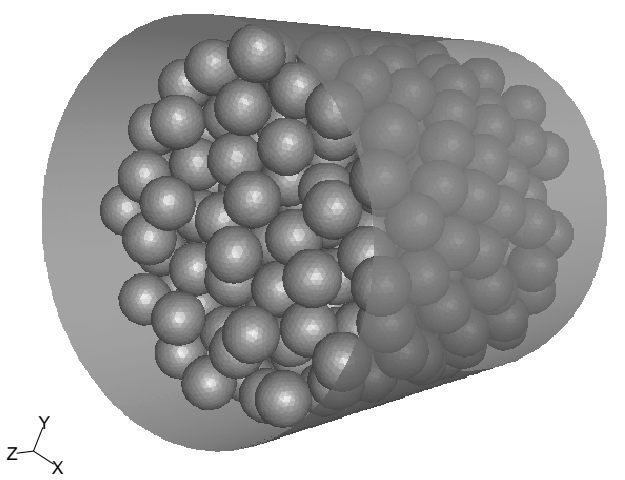

Grid (Time $=8.1700 \mathrm{e}-06)$

FLUENT 6.3 (3d, pbns, sstkw, unsteady)

Figure 2: View of the bed of spheres used for the calculation.

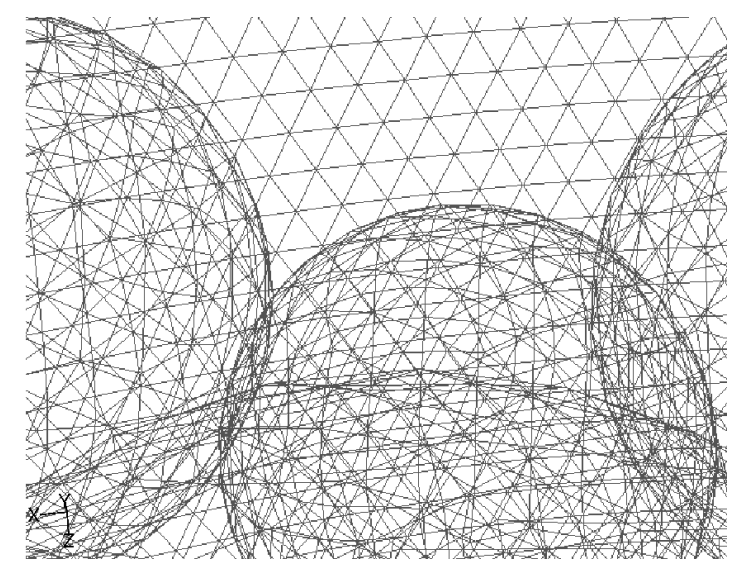

Figure 3: Detail of geometry and mesh at the top of the bed 


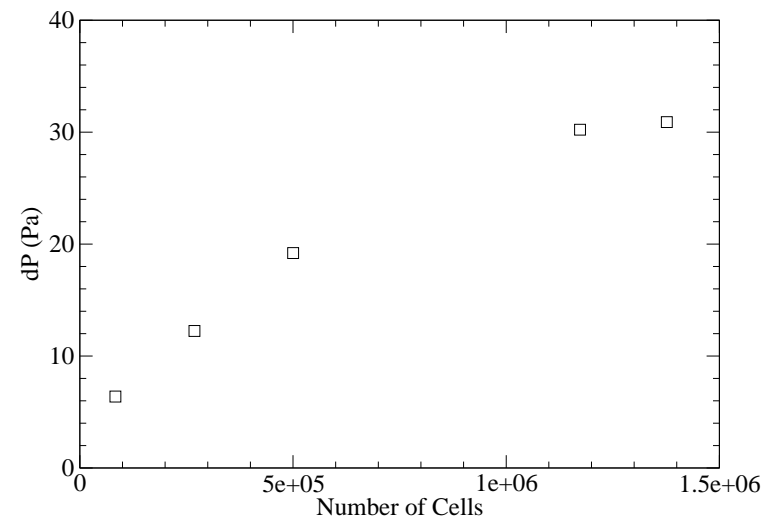

Figure 4: Mesh convergence study; calculated pressure drop vs. number of cells for a range of different meshes.

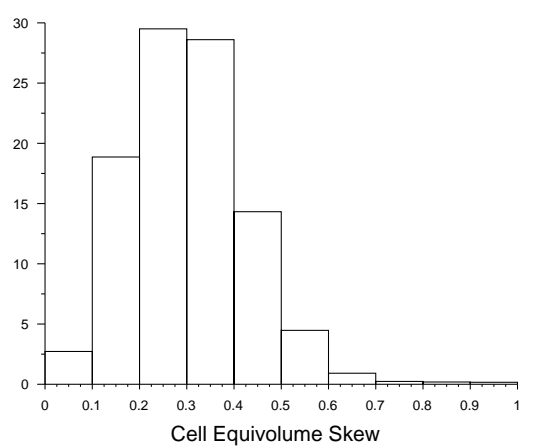

Histogram of Cell Equivolume Skew (Time=3.2700e-05)

FLUENT 6.3 (3d, pbns, skw, 22, 2009

Figure 5: Histogram showing distribution of cell equivolume skew values. 


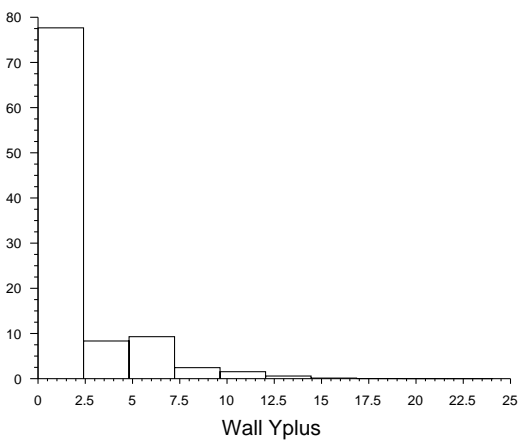

Histogram of Wall Yplus (Time $=3.2700 \mathrm{e}-05)$ Mar 22, 2009
FLUENT 6.3 (3d, pbns, skw, unsteady)

Figure 6: Histogram showing distribution of yplus $\left(y^{+}\right)$values for the wall region. 
a.

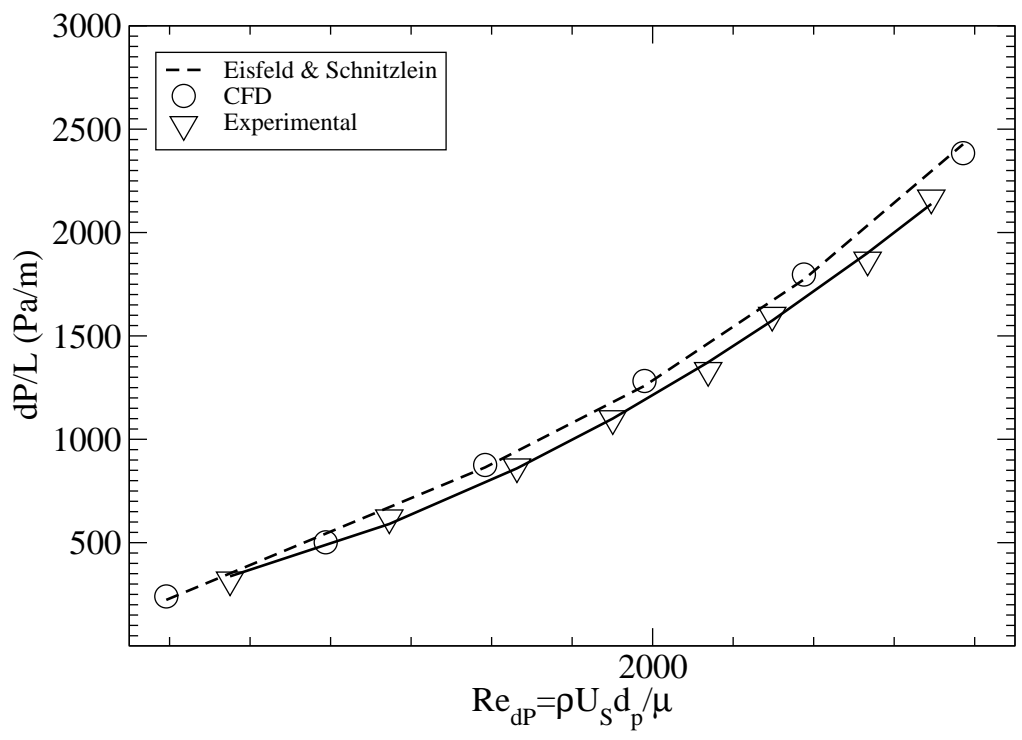

b.

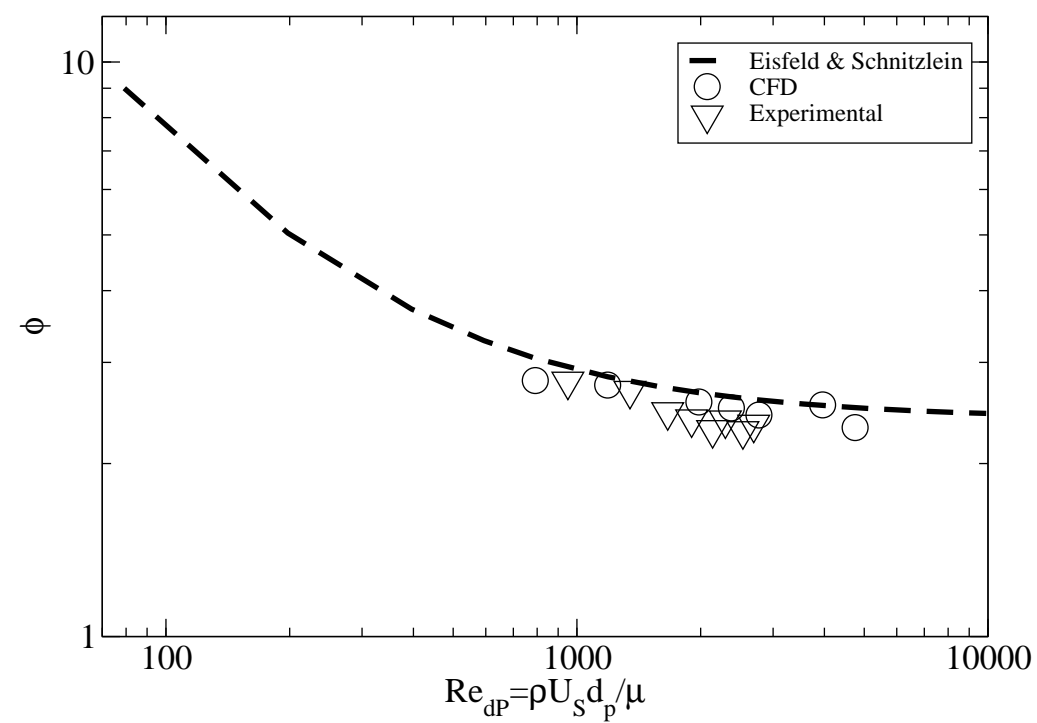

26

Figure 7: a. Pressure drop per unit length as a function of the particle Reynolds number $\left(R e_{p}\right)$. b. Dimensionless pressure drop $(\phi)$ as a function of particle Reynolds number $\left(R e_{p}\right)$. 
a.

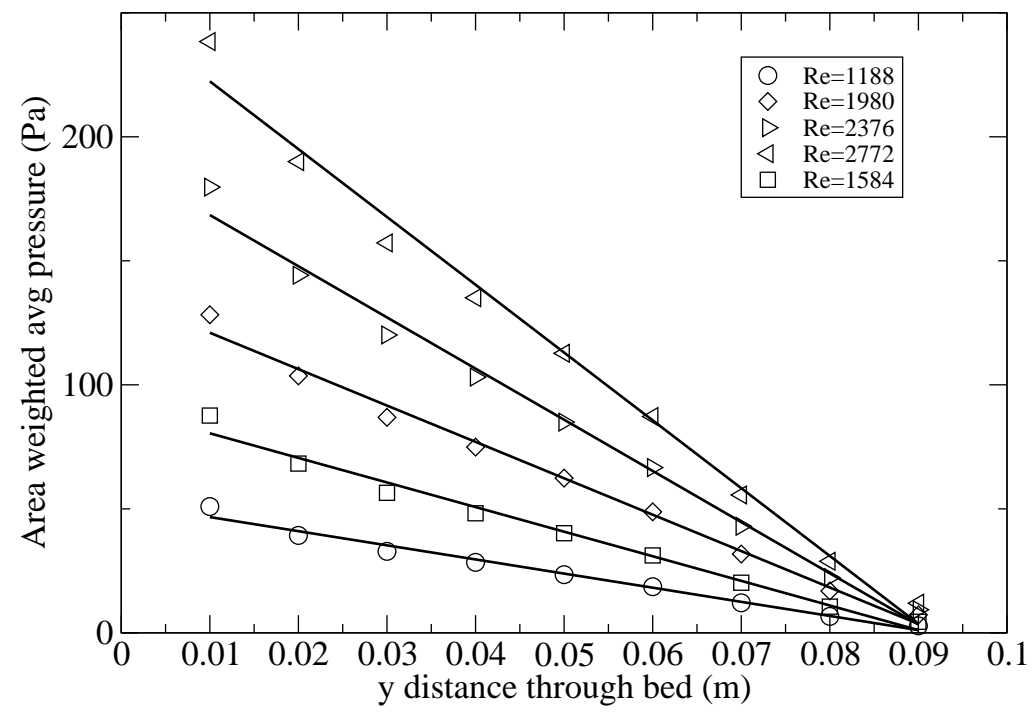

b.

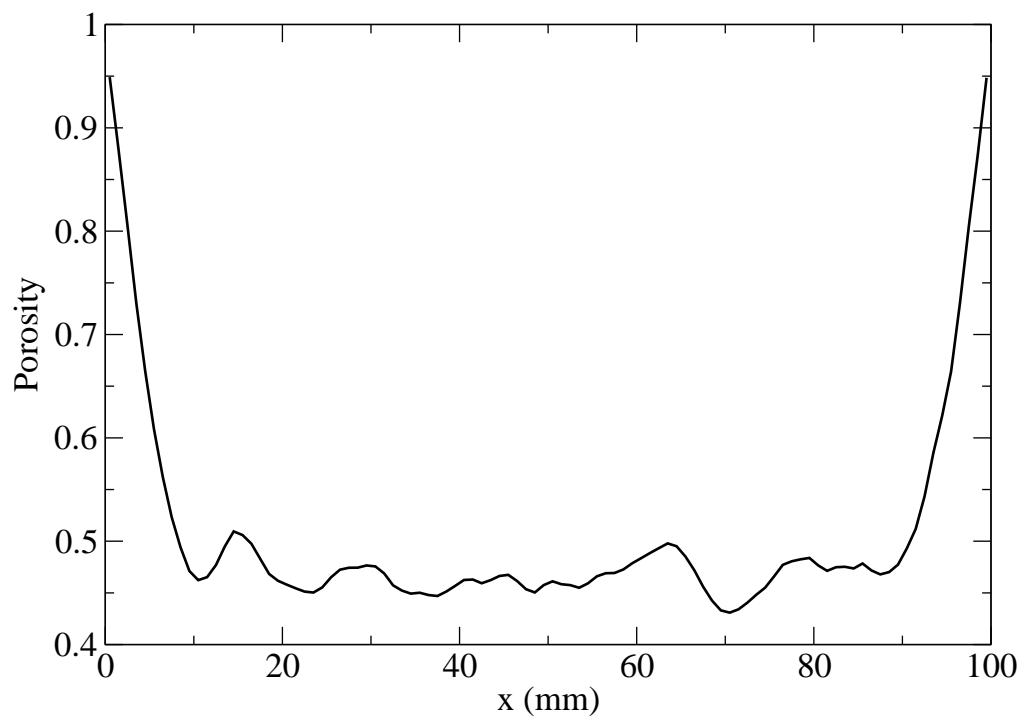

27

Figure 8: a. Average pressure across the x-plane of the bed at y-intervals of $0.01 \mathrm{~m}$. b. Porosity as a function of distance across the $\mathrm{x}$-plane of packed bed. 
a.

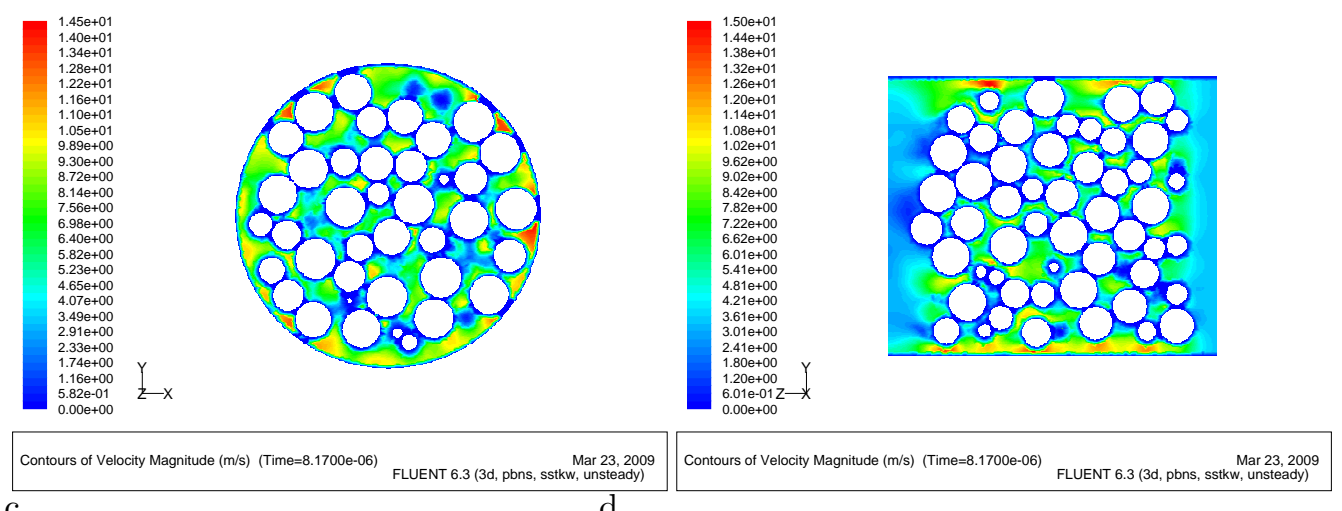

c.

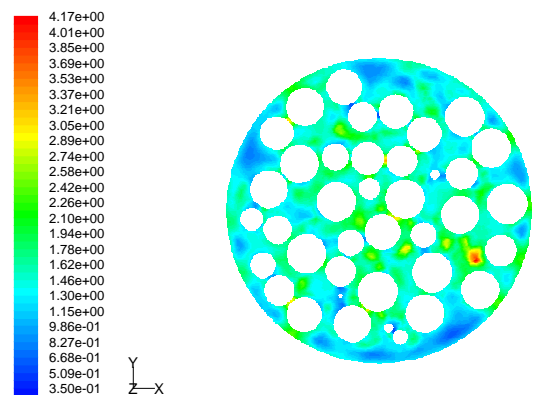

b.

d.

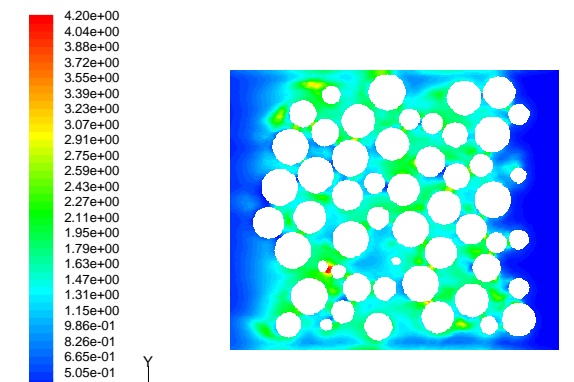

Contours of Turbulent Kinetic Energy (k) (m2/s2) (Time=8.1700e-06)
FLUENT 6.3 (3d, pbns, sstkw, unsteady)

Mar 23, 2009
kw, unsteady)

Figure 9: Contours of velocity magnitude for $14 \mathrm{~mm}$ spheres (a,b), contours of turbulent kinetic energy $(c, d)$. Flow is in the $+\mathrm{z}$ direction. 